\title{
Encyclopedia of autoimmune pancreatitis: this is all we need
}

\author{
Eizaburo Ohno ${ }^{1} \cdot$ Yoshiki Hirooka²
}

Received: 14 September 2021 / Accepted: 25 September 2021

(c) The Author(s), under exclusive licence to The Japan Society of Ultrasonics in Medicine 2021

$\begin{array}{ll}\text { Abbreviations } \\ \text { AIP } & \text { Autoimmune pancreatitis } \\ \text { OOI } & \text { Other organ involvement } \\ \text { ICDC } & \text { International consensus diagnostic criteria } \\ \text { TUS } & \text { Transabdominal ultrasonography } \\ \text { EUS } & \text { Endoscopic ultrasonography } \\ \text { CE-US } & \begin{array}{l}\text { Contrast-enhanced ultrasonography } \\ \text { CT }\end{array} \\ \text { Computed tomography } \\ \text { MRI } & \text { Magnetic resonance imaging } \\ \text { ERCP } & \begin{array}{l}\text { Endoscopic retrograde } \\ \text { cholangiopancreatography }\end{array} \\ \text { EUS-FNB } & \text { EUS-guided fine-needle biopsy } \\ \text { EUS-FNA } & \text { EUS-guided fine-needle aspiration } \\ \text { EUS-EG } & \text { EUS elastography } \\ \text { CEH-EUS } & \text { Contrast-enhanced harmonic EUS } \\ \text { MRCP } & \begin{array}{l}\text { Magnetic resonance } \\ \text { cholangiopancreatography }\end{array} \\ \text { IDUS } & \text { Intraductal ultrasonography }\end{array}$

\section{Introduction}

Autoimmune pancreatitis (AIP) is a pancreatic inflammatory disease characterized by pancreatic swelling that requires differentiation from pancreatic cancer. In Japan, the majority of patients are type 1 AIP, which is diagnosed as a partial phenotype of IgG4-related disease, but there are also patients with type 2 AIP, which is not associated with increased serum IgG4. It has also been reported that IgG4related disease is associated with various inflammatory changes not only in the pancreas but also in multiple organs as other organ involvement (OOI). Diagnostic criteria of AIP were published in the International Consensus Diagnostic

Eizaburo Ohno

eono@med.nagoya-u.ac.jp

1 Department of Gastroenterology and Hepatology, Nagoya University Graduate School of Medicine, Nagoya, Japan

2 Department of Gastroenterology and Hepatology, Fujita Health University, Toyoake, Japan
Criteria (ICDC) and the clinical diagnostic criteria published by the Japanese Pancreas Society in 2018 (JPS2018), but the pathophysiology and diagnostic criteria of AIP are complicated $[1,2]$.

Transabdominal ultrasonography (TUS) and endoscopic ultrasonography (EUS) are indispensable diagnostic modalities for AIP in terms of screening of AIP patients, differential diagnosis from pancreatic tumors such as pancreatic cancer, and a method of tissue acquisition by EUS-guided fine-needle biopsy (EUS-FNB). In this special issue, we have topics not only about the characteristics of ultrasonographic findings on TUS and EUS for AIP but also radiological diagnosis using computed tomography (CT)/magnetic resonance imaging (MRI) and endoscopic retrograde cholangiopancreatography (ERCP). Furthermore, we asked an expert pathologist to provide a detailed commentary on the pathophysiology of AIP. These topics provide all of the current status of AIP diagnosis and are surely helpful for a deeper understanding of the pathophysiology of AIP, especially for sonographers and endosonographers.

\section{Ultrasonography and endoscopic ultrasonography}

TUS is the most commonly used screening method for pancreatic diseases, and understanding the characteristic ultrasonographic findings of AIP is essential for subsequent detailed examinations. Okaniwa [3] outlined the characteristics of AIP using B-mode ultrasound in TUS. In addition, this review introduced new findings of blood flow diagnosis using contrast-enhanced ultrasonography (CE-US) and measurement of tissue elasticity diagnosis using ultrasonic elastography. It also provided the ultrasonographic characteristics of extra-pancreatic lesions as $\mathrm{OOI}$ in patients with IgG4-related disease.

Compared to TUS, EUS enables detailed observation with high spatial resolution. Ishikawa et al. [4] outlined the characteristics of the imaging of EUS including B-mode images, EUS elastography (EUS-EG), and contrastenhanced harmonic EUS (CEH-EUS) in AIP diagnosis. In 
particular, EUS-EG, software for measuring tissue elasticity, introduced the possibility of evaluating the pathophysiology of AIP as well as the differential diagnosis of other pancreatic diseases.

The gold standard for diagnosis of AIP is the characteristic pathological findings. In recent years, the number of cases of surgical resection for AIP has decreased, and the specimens used for histopathological diagnosis are mainly obtained by EUS-guided fine-needle aspiration or biopsy (EUS-FNA/B). To make a reliable pathological diagnosis of AIP, it is essential to have a sufficient amount of tissue that can be appropriate for immunohistochemical staining. Kanno et al. [5] explained the procedures of tissue collection by EUS-FNA/B and the recent development of biopsy needles for the purpose of better tissue collection. And they introduced tips on how to handle tissue samples to enhance the diagnostic ability with limited biopsy specimens.

\section{Radiological findings of AIP and IgG4-related sclerosing cholangitis}

According to the diagnostic criteria in the ICDC [1] and JPS2018 [2], diagnostic imaging using CT/MRI (MRCP) is the main axis of imaging diagnosis. Ogawa and Takehara [6] comprehensively described the characteristics of pancreatic and extra-pancreatic findings of AIP in CT and MRI imaging. They also touched on the usefulness of a new diagnostic imaging method for MRI in the future diagnosis of AIP.

One of the imaging characteristics of AIP in the ICDC is pancreatic duct narrowing findings on endoscopic retrograde pancreatography (ERP). In recent years, pancreatic duct findings have tended to replace MRCP findings, but they are still important findings for distinguishing AIP from pancreatic cancer. In addition, type 1 AIP may involve bile duct lesions in addition to pancreatic lesions. These lesions, which are called IgG4-related sclerosing cholangitis (IgG4$\mathrm{SC}$ ), are associated with specific narrowing or stenosis of the bile duct, and require differentiation from biliary malignancy or primary sclerosing cholangitis. Naito et al. [7] explained in detail the characteristics of imaging features of IgG4SC on ERCP and intraluminal ultrasonography (IDUS) and showed the points of differentiation from other biliary diseases.

\section{Pathological findings}

The gold standard in the diagnosis of AIP is based on pathological findings. Notohara [8] described in detail the pathological findings and characteristics of AIP. Understanding the clinical features and imaging findings of AIP in relation to the corresponding pathological features contributes to a deeper and more accurate understanding of the pathophysiology of AIP. He also explains the limitations of pathological diagnosis based on the specimens collected by EUS-FNA/B in clinical practice from the standpoint of a pathologist.

\section{Conclusion}

We asked the authors, who are experts in each field, to review the latest information on AIP diagnosis. This special issue covers the current status of AIP diagnosis, and this will definitely help us in our daily practice, education, and when writing papers of our research.

\section{Postscript}

Each paper may use different terminology for the appearance and findings of the same condition on US and EUS. In this special issue, the original text has been retained to respect the originality of each author. This does not diminish the academic value of each paper or of this special issue.

\section{References}

1. Shimosegawa T, Chari ST, Frulloni L, et al. International Association of Pancreatology. International consensus diagnostic criteria for autoimmune pancreatitis: guidelines of the International Association of Pancreatology. Pancreas. 2011;40:352-8.

2. Kawa S, Kamisawa T, Notohara K, et al. Japanese clinical diagnostic criteria for autoimmune pancreatitis, 2018: revision of Japanese clinical diagnostic criteria for autoimmune pancreatitis, 2011. Pancreas. 2020;49:e13-4.

3. Okaniwa S. Role of transabdominal ultrasound in the diagnosis of autoimmune pancreatitis. J Med Ultrason. 2021. https://doi.org/10. 1007/s10396-021-01133-y.

4. Ishikawa $\mathrm{T}$, Kawashima $\mathrm{H}$, Ohno E, et al. Imaging diagnosis of autoimmune pancreatitis using endoscopic ultrasonography. J Med Ultrason. 2021. https://doi.org/10.1007/s10396-021-01143-w.

5. Kanno A, Tamada K, Fukushima N, et al. Endoscopic ultrasoundguided tissue acquisition for the histopathological diagnosis of autoimmune pancreatitis. J Med Ultrason. 2021. https://doi.org/10.1007/ s10396-021-01144-9.

6. Ogawa H, Takehara Y, Naganawa S. Imaging diagnosis of autoimmune pancreatitis: computed tomography and magnetic resonance imaging. J Med Ultrason. 2021. https://doi.org/10.1007/ s10396-021-01145-8.

7. Naitoh I, Nakazawa T. Endoscopic retrograde cholangiopancreatography and intraductal ultrasonography in the diagnosis of autoimmune pancreatitis and IgG4-related sclerosing cholangitis. J Med Ultrason. 2021. https://doi.org/10.1007/s10396-021-01114-1.

8. Notohara K. Histopathological features of autoimmune pancreatitis and IgG4-related sclerosing cholangitis with a correlation with imaging findings. J Med Ultrason. 2021. https://doi.org/10.1007/ s10396-021-01148-5.

Publisher's Note Springer Nature remains neutral with regard to jurisdictional claims in published maps and institutional affiliations. 